\title{
Effects of Midfrontal Brain Stimulation on Sustained Attention
}

\author{
Martine R. van Schouwenburg ${ }^{1,2}$ • Ilja G. Sligte ${ }^{1,2}$ - Michael R. Giffin ${ }^{1,2}$ • Franziska Günther ${ }^{1,2}$ • Dirk Koster ${ }^{1,2}$. \\ Floortje S. Spronkers ${ }^{1,2} \cdot$ Anna $\operatorname{Vos}^{1,2} \cdot$ Heleen A. Slagter ${ }^{1,2,3}$
}

Received: 7 February 2020 / Accepted: 7 June 2020 / Published online: 17 June 2020

(C) The Author(s) 2020

\begin{abstract}
Sustained attention is defined as the ability to maintain attention over longer periods of time, which typically declines with time on task (i.e., the vigilance decrement). Previous studies have suggested an important role for the dorsomedial prefrontal cortex (mPFC) in sustained attention. In two experiments, we aimed to enhance sustained attention by applying transcranial electrical current stimulation over the mPFC during a sustained attention task. In the first experiment, we applied transcranial direct current stimulation (tDCS) in a between-subject design $(n=97)$ : participants received either anodal, cathodal, or sham stimulation. Contrary to our prediction, we found no effect of stimulation on the vigilance decrement. In the second experiment, participants received theta and alpha transcranial alternating current stimulation (tACS) in two separate sessions $(n=47$, within-subject design). Here, we found a frequency-dependent effect on the vigilance decrement, such that contrary to our expectation, participants' performance over time became worse after theta compared with alpha stimulation. However, this result needs to be interpreted with caution given that this effect could be driven by differential side effects between the two stimulation frequencies. To conclude, across two studies, we were not able to reduce the vigilant decrement using tDCS or theta tACS.
\end{abstract}

Keywords Vigilance $\cdot$ Sustained attention $\cdot$ Prefrontal cortex $\cdot$ tDCS $\cdot$ tACS

\section{Introduction}

Sustained attention, the ability to filter incoming sensory information and maintain attention to this information for longer time periods, is an important aspect of cognitive functioning. Most people will experience that it is much easier to maintain attention over a longer period of time when in an engaging environment (for example driving a car in a city), compared with when they find themselves in a rather uneventful environment (driving a car on an empty highway). Indeed, research has shown large decrements in performance over time on sustained attention tasks that require participants to monitor and detect infrequent targets in a stream of non-targets

Heleen A. Slagter

h.a.slagter@vu.nl

1 Department of Psychology, University of Amsterdam, Nieuwe Achtergracht 129B, Amsterdam, the Netherlands

2 Amsterdam Brain and Cognition (ABC), University of Amsterdam, Nieuwe Achtergracht 129B, Amsterdam, the Netherlands

3 Department of Applied and Experimental Psychology, Vrije Universiteit Amsterdam, Van der Boechorststraat 7, 1081 BT Amsterdam, the Netherlands
(Warm et al. 2008; Robertson and O'Connell 2010). Yet, sustained attention is crucial in daily life and in particular in jobs such as air traffic control, lifeguarding, or inspection for quality control since in these jobs a drop in attention could have large detrimental consequences. In the current study, we investigated if it is possible to improve sustained attention using brain stimulation. Specifically, we tested whether transcranial electrical stimulation can prevent or reduce the timedependent vigilance decrement.

According to Stuss et al., sustained attention relies on four distinct processes (Norman and Shallice 1986; Stuss et al. 1995). Activation of the task-relevant schema has to be monitored (1), and if activity drops below a certain threshold, activity of the task-relevant schema has to be reactivated (2). In addition, to reduce interference, conflicting schemata have to be inhibited (3). Finally, a higher-order process monitors task performance and takes action if performance drops (4) (through reactivation of the task-relevant schema and/or inhibition of conflicting task schemata).

The question then arises which of these processes fails during the vigilance decrement. Theoretical frameworks have attributed the time-dependent drop in performance to three (not mutually exclusive) causes: enhanced mind wandering, resource depletion, and/or reduced motivation. Some have 
suggested that sustained attention tasks are just not engaging enough, leading to mind-wandering (Robertson et al. 1997; Smallwood and Schooler 2006). This means that people fail to inhibit conflicting schemata and are distracted from the main task leading to the decrement in performance (Langner and Eickhoff 2013). Others have suggested that after sustaining attention for a certain period of time, attentional resources are depleted, leading to an inability to reactivate the taskrelevant schema (Helton and Warm 2008). There is much empirical evidence that supports resource depletion, and this "mental fatigue" account is in line with the subjective experience of the task becoming harder over time (Warm et al. 2008; Grier et al. 2003). However, studies have also shown that social comparison or monetary rewards can improve performance (Boksem et al. 2006; Lorist et al. 2009; Bonnefond et al. 2011; Hopstaken et al. 2015). For example, a recent study by our lab showed that the sustained attention decrement can be partly resolved by an unexpected monetary incentive (Reteig et al. 2019). This suggests that resource depletion cannot fully account for the performance decrement, but that motivation is also crucial for maintaining vigilance. This is in line with motivational control theories by Robert and Hockey (1997). Others have combined the resource depletion and motivational control accounts into hybrid models that weigh the potential reward outcome of an action against the anticipated energy expenditure (Boksem and Tops 2008; Christie and Schrater 2015). Taken together, it seems likely that multiple factors interact to cause the vigilance decrement.

In an effort to better understand the underlying causes of the vigilance decrement, researchers have turned to neuroimaging. In a recent meta-analysis, Langner and Eickhoff (2013) identified a network of brain regions that plays a key role in maintaining sustained attention. This network comprised a large cluster spanning the presupplementary motor area (pre-SMA), midcingulate cortex, extending into more anterior medial prefrontal cortex (PFC), and clusters in midlateral and ventrolateral PFC, anterior insula, parietal areas, and subcortical structures. The authors next built a hierarchical model of how these different network nodes might interact and their putative role in sustained attention (Langner and Eickhoff 2013). In this model, the large frontal cluster (which we will from now on refer to as medial PFC (mPFC)), plays a central role both due to its position at the top of the hierarchy and its role in performance monitoring and task-set (re-)energizing. This view corroborates a large body of evidence that links the mPFC with error monitoring and subsequent adjustments in attentional control (for review, see (Ridderinkhof et al. 2004)). Improper functioning of the mPFC could lead to the inability to activate the taskrelevant schema and thereby contribute to the vigilance decrement. Moreover, mPFC activity increases with time-on-task (Langner and Eickhoff 2013; Boksem et al. 2005; Wascher et al. 2014), which is thought to reflect enhanced effort to maintain attentional focus on the task (Clayton et al. 2015). In many of these studies, mPFC activity was measured through power in the theta band. Indeed, theta oscillations seem to be the lingua franca of the mPFC and are strongly linked to cognitive control processes (Cavanagh and Frank 2014). In sum, midfrontal theta oscillations seem to play an important role in our ability to maintain sustained attention over time.

The goal of our study was to determine if electrical stimulation over the mPFC can improve the ability to sustain attention over time. To this end, we performed two experiments that used transcranial electrical stimulation. In experiment 1 , we applied transcranial direct current stimulation (tDCS) over the mPFC. In this technique, a small electrical current is passed between two electrodes, which is thought to depolarize (anodal stimulation) or hyperpolarize (cathodal stimulation) the underlying neural tissue (Nitsche and Paulus 2011). We hypothesized that anodal stimulation over the mPFC would increase cortical activity in this region, resulting in improved sustained attention. This hypothesis also seems justified given previous work that has shown that anodal midfrontal tDCS can increase midfrontal theta activity (Miller et al. 2015). Conversely, we predicted that cathodal stimulation over the mPFC would decrease cortical excitability, resulting in reduced sustained attention. In experiment 2, we applied transcranial alternating current stimulation (tACS) over the mPFC. tACS is similar to tDCS, except that the direction of the current alternates at a certain frequency. This is thought to entrain neurons in the underlying neural tissue to the stimulated frequency (Herrmann et al. 2013). By stimulating the mPFC in theta frequency, we expected to improve sustained attention.

\section{Materials and Methods}

\section{Experiment 1}

\section{Participants}

One hundred one healthy participants were included in the experiment and were tested in a double-blind, randomized, between-subject design. They were selected based on the following criteria; no epilepsy or (family) history of an epileptic seizure, no neurological disorders, no history of stroke or other forms of brain damage, no history of a severe concussion, no (history of) meningitis, no use of psychoactive substances, no cardiac pacemakers or other implanted medical devices, no metal anywhere in the head, no albinism, not pregnant, not recently fainted, no recent panic attack, no multiple sclerosis, no skin abnormalities on the head, and no color blindness.

All participants had normal or corrected-to-normal vision, and all but three participants were right handed. Participants were instructed to abstain from alcohol and non-prescriptive medication and illicit substances $24 \mathrm{~h}$ 
prior to the experiment. If they failed to follow these instructions, they were excluded from participation.

We aimed to include at least 30 subjects per group in our final sample, i.e., double the number of subjects that previous studies included in a recent review paper (see Table 4 in (Reteig et al. 2017)) and that used a between-subject design to study the effect of transcranial electrical stimulation on sustained attention performance on average included per group $\left(n_{\text {avg }}=15.6\right)$. Two participants decided not to continue with the experiment after the trial stimulation was applied. In addition, two participants did not complete the experiment (one was asked to leave because the participant was not doing the task, and the other participant accidently aborted the task). The remaining 97 participants ( 71 females) had a mean age of 22.3 years $(\mathrm{sd}=2.7)$ and were randomly assigned to one of three stimulation groups: anodal (33 subjects, mean age 21.9 years, sd 2.9, 23 females), cathodal (34 subjects, mean age 22.6 years, sd 2.3, 25 females), or sham ( 30 subjects, mean age 22.4 years, sd 2.9, 23 females).

The protocol was approved by the Social Sciences ethical committee of the University of Amsterdam. The methods were carried out in accordance with the relevant guidelines and regulations. All participants gave written informed consent in accordance with the Declaration of Helsinki and received course credits or a monetary reward in exchange for participation.

\section{Paradigm}

Participants performed a sustained attention task adapted from MacLean et al. (2009). In this task (Fig. 1), participants had to discriminate between a target (short line/33\% of stimuli) and non-target (long line/67\% of stimuli) and respond to the target stimuli by clicking the left mouse button with their right index finger. Throughout the experiment, participants were told to fixate on a yellow fixation dot in the middle of the screen.
Between stimuli, a gray mask, composed of stacked short lines, was presented to prevent an after-image of the stimuli, which would otherwise allow participants to easily compare line lengths across trials. This mask changed on each presentation to prevent participants from comparing stimulus line length to the mask. Specifically, on each presentation, the lines that comprised the mask were vertically repositioned by a random amount ( -4 to +4 pixels). Stimuli were on the screen for $150 \mathrm{~ms}$, while the interstimulus interval (during which the mask was presented) was randomly chosen on each trial between 1350 and $2350 \mathrm{~ms}$.

Visual stimuli were presented using Presentation software (Neurobehavioral Systems) on a 24-in. monitor with a $1920 \times$ 1080 resolution. Non-targets and targets had a width of 2 pixels. The height of non-targets (long line) was consistent across participants (122 pixels), while the height of the target (short line) was defined individually to balance task difficulty across participants (average $=93.4$ pixels, $\mathrm{SD}=13.0$, range 20-112).

Individual target line length was determined with a Parameter Estimation by Sequential Testing (PEST) procedure (Taylor and Creelman 1967; MacLean et al. 2009). During this calibration procedure, participants performed the sustained attention task described above while the length of the target line was adaptively changed according to a 3-down, 1-up staircase until performance stabilized at $80 \%$ accuracy. To help participants to learn the task, auditory feedback was provided following correct identification of a target and following a miss or a false alarm.

\section{Study Design}

The study followed a double-blind randomized betweenparticipant design. At the start of the session, participants were checked for exclusion criteria and asked about drug and alcohol use in the last $24 \mathrm{~h}$. Next, participants were seated $57 \mathrm{~cm}$ from the monitor, received task instructions, and performed
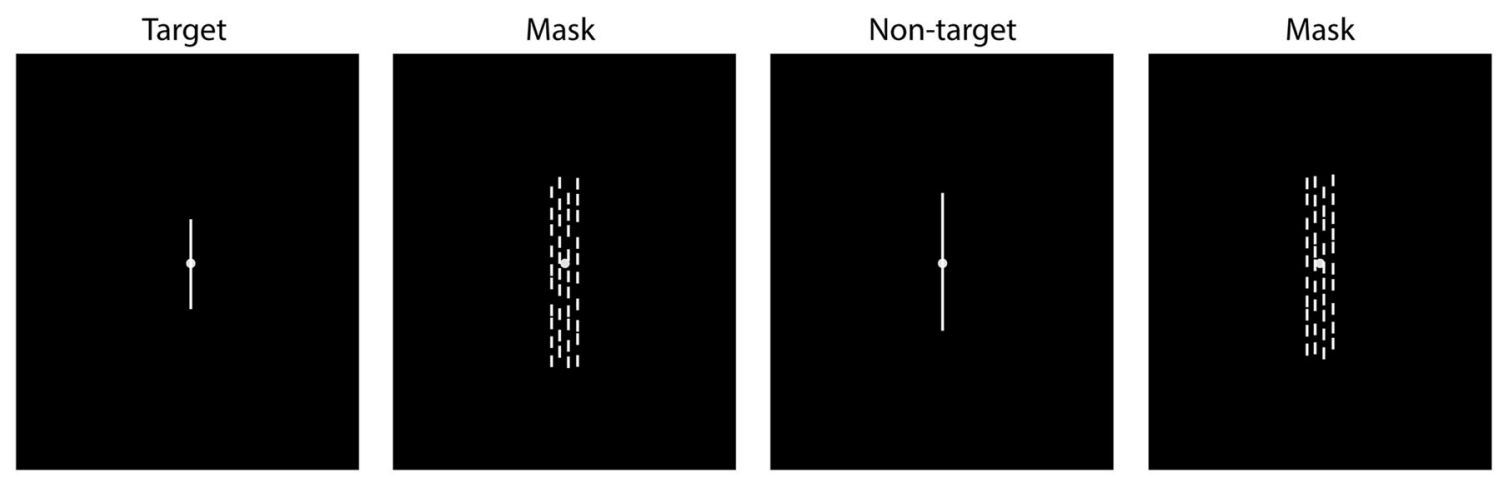

Time

Fig. 1 In both experiments, participants performed a sustained attention task in which they had to respond to an infrequent target stimulus (short line), and withhold responses to the non-target (long line). Presentation of stimuli (150 ms) was interleaved with the presentation of masks (variable time interval, see main text) 
the PEST procedure. Completion of the PEST procedure lasted approximately $10 \mathrm{~min}$, but exact length varied between subjects due to the dynamic stopping rule used by the PEST titration. Upon completion of the PEST, stimulation electrodes were attached and participants were informed about any possible side effects that could occur. Participants then received $1 \mathrm{~min}$ of trial stimulation ( $5 \mathrm{~s}$ ramp up, $60 \mathrm{~s}$ stimulation, $5 \mathrm{~s}$ ramp down). Participants were asked about their experience both during and after trial stimulation. If a subject reported uncomfortable side effects and/or impedance was above $25 \mathrm{~m} \Omega$, the setup was adjusted to lower impedance. In this case, trial stimulation was applied again to ensure that participants felt comfortable with the stimulation. After trial stimulation, participants performed the sustained attention task for a total of 60 min divided over three blocks of $20 \mathrm{~min}$. In the second of these 20-min blocks, stimulation was applied. This allowed us to examine if tDCS could revert or prevent the vigilance decrement from further developing. Before the start of each block, and at the end of the experiment, participants answered two questions about how motivated they were and how much aversion they felt toward the task by moving an arrow over an 1100-pixels-wide scale by scrolling the mouse wheel. Stimulation electrodes were removed after the second 20-min block. After the experiment ended, the participants filled out a questionnaire about possible side effects of the stimulation, as well as the perceived effect of stimulation on task performance. Side effects were scored on a linear scale from 0 to 100 , with a score of zero representing "not at all applicable to me" and a score of 100 representing "completely applicable to me." The perceived effect of stimulation on task performance was rated as worse, similar, or better performance compared with the blocks without stimulation. Ratings for 10 possible side effects were provided: headache, neck pain, nausea, muscle contractions, stinging sensations, burning sensations, tiredness, mood changes, uncomfortable feeling, and phosphenes.

\section{tDCS}

tDCS (NeuroConn DC-Stimulator MR, NeuroConn, Germany) was administered via two rubber electrodes. Electrodes were placed in saline-soaked sponges and attached to the head via straps and tape. One electrode $\left(9 \mathrm{~cm}^{2}\right)$ was placed between electrode locations $\mathrm{FCz}$ and $\mathrm{Cz}$ on the 10 20 international EEG system, and a second electrode $(35 \mathrm{~cm} 2)$ was placed on the left cheek (van Driel et al. 2015). Stimulation intensity was set to $1 \mathrm{~mA}$ (current density of $0.11 \mathrm{~mA} / \mathrm{cm}^{2}$ at $\mathrm{mPFC}$ electrode). During the anodal stimulation condition, the anode was placed at $\mathrm{Cz} / \mathrm{FCz}$ and the cathode at the cheek, while the opposite was true for the cathode condition. Stimulation was ramped up for $60 \mathrm{~s}$ and, after the 20-min stimulation period, ramped down again for $60 \mathrm{~s}$. In the sham group, half of the participants received "anodal" sham stimulation, while the other half received "cathodal" sham stimulation. In this control condition, stimulation was ramped up for $60 \mathrm{~s}$ and then ramped down again over $60 \mathrm{~s}$. Experimenters were blinded to stimulation condition, as the stimulation settings were programmed by a colleague beforehand and they could not see the settings.

\section{Data Analysis}

Data were analyzed using Matlab (The MathWorks, Inc.), SPSS (IBM SPSS Statistics, version 23.0), and JASP (version 0.12.2) (Team 2019). Per 20-min block (pre, during and post stimulation), we determined the hit rate and false alarm rate and subsequently calculated the non-parametric perceptual sensitivity measure $\mathrm{A}^{\prime}$ as formulated by Stanislaw and Todorov (Stanislaw and Todorov 1999) (and implemented cf. (Reteig et al. 2019; MacLean et al. 2009; Slagter et al. 2016)). $\mathrm{A}^{\prime}$ is based on signal detection theory and indicates a subject's ability to discriminate between targets and non-targets. Values typically range from 0.5 (subjects cannot distinguish targets from noise) to 1 (subjects distinguish all targets from noise) (Stanislaw and Todorov 1999). In addition, we calculated the average reaction time per block.

Data were submitted to repeated measures ANOVAs with the between-subject factor stimulation condition (sham vs. cathodal vs. anodal) and the within-subject factor time (pre stimulation vs. during stimulation vs. post stimulation). Post hoc paired $t$ tests were used when significant results were found. In addition, ANCOVAs were used in order to assess whether reported side effects and target line length differed across stimulation conditions. When possible, we also conducted Bayesian variants of these statistical tests, to quantify the strength of evidence for or against an effect. We report the $\mathrm{BF}_{10}$ when the alternative hypothesis was tested, and $\mathrm{BF}_{01}$ when the null hypothesis was tested. BF values reported for interaction effects indicate the extent to which there is more/ less evidence for the model that includes the interaction effect (next to the main effects) vs. the model that only includes the main effects.

\section{Experiment 2}

\section{Participants}

The methods were the same as in experiment 1 except for the following changes. Fifty-five healthy participants were included in the experiment and were tested in a randomized, within-subject design. In addition to the exclusion criteria mentioned for experiment 1 , we also screened for spondylosis, scoliosis, arthritis, and frequent occurrences of dizziness or headaches. The sample size tested was based on previous studies using a within-subject design to study the effects of transcranial electrical stimulation on sustained attention task 
performance included a recent review (see Table 4 in (Reteig et al. 2017)) and chosen to be twice as large as the average sample size $\left(n_{\text {avg }}=27.6\right)$ used in these previous studies.

All participants were right-handed and had normal or corrected-to-normal vision. Three participants decided not to continue with the experiment after the trial stimulation was applied, and three participants were eliminated due to technical reasons (stimulation was automatically aborted at some point during the stimulation block due to high impedance levels). One participant drank alcohol within the $24 \mathrm{~h}$ before the experiment and was therefore excluded. Finally, one participant dropped out after the first session. The remaining 47 participants (34 female) had a mean age of 21.5 years $(\mathrm{SD}=2.6)$.

\section{Paradigm}

The paradigm was adapted in two ways. First, the target/nontarget ratio was adapted, so that now $25 \%$ of stimuli was a target (compared with $33 \%$ in experiment 1). Second, the inter-stimulus interval lasted a minimum of $1550 \mathrm{~ms}$ and a maximum of $2150 \mathrm{~ms}$ (compared with $1350-2350 \mathrm{~ms}$ in experiment 1). Again, the height of the target (short line) was defined individually, on each session, to balance task difficulty across participants (average over two sessions $=95.1$ pixels, $\mathrm{SD}=8.3$, range $55-111$ ).

\section{Study Design}

In contrast to the first experiment, we used a within-subject design in the second experiment to enhance statistical power. Participants participated in two sessions separated by 1 week (except for 6 participants in which the sessions were separated somewhere between 6 and 15 days). The two sessions were similar except for the frequency of the applied tACS stimulation. The order of the stimulation condition was randomized, and both participants and the experimenter were blind to the stimulation condition (double-blind design). The general outline of the sessions was similar to the one in the first experiment with the exception of the following: (1) trial stimulation consisted of $1 \mathrm{~min}$ of alpha stimulation (10 s ramp up, $30 \mathrm{~s}$ stimulation, $10 \mathrm{~s}$ ramp down), (2) participants performed the sustained attention task for a total of $50 \mathrm{~min}(15 \mathrm{~min}$ before and after stimulation, and 20 min during stimulation), and (3) side effects were scored on a five-point scale with a score of one representing "not at all applicable to me" and a score of five representing "completely applicable to me." In addition, we tried to minimize breaks during the task as much as possible. Therefore, the questions about motivation and aversion were eliminated from the design and we did not remove the stimulation electrodes until after the end of the experiment. Thus, there were no pauses during the experiment except for the time required to start stimulation in between block one and two, which lasted approximately $1 \mathrm{~min}$. No interaction with the participants took place during this minute to minimize the duration of the task break. Participants were seated approximately $65 \mathrm{~cm}$ from the screen.

\section{tACS}

One milliampere of tACS (NeuroConn DC-Stimulator MR, NeuroConn, Germany) was administered via three rubber electrodes. Electrodes were placed inside saline-soaked sponges and attached to the head via straps and tape. The target electrode $\left(9 \mathrm{~cm}^{2}\right)$ was placed between electrode locations $\mathrm{FCz}$ and $\mathrm{Cz}$ on the 10-20 international EEG system as in experiment 1 . Two reference electrodes $\left(35 \mathrm{~cm}^{2}\right)$ were placed on the cheeks. This setup has previously been used by van Driel et al. (2015) and is aimed to direct current flow through the mPFC. Stimulation intensity ranged between -1 and + $1 \mathrm{~mA}$ and was set to sinusoidal (current density of $0.11 \mathrm{~mA} /$ $\mathrm{cm}^{2}$ at $\mathrm{mPFC}$ electrode). Fading in and out of the stimulation lasted for $10 \mathrm{~s}$ each.

For the active condition, stimulation frequency was set to $4 \mathrm{~Hz}$ (theta band). During the control condition and trial stimulation, $10 \mathrm{~Hz}$ (alpha band) tACS was applied. Alpha band stimulation instead of sham was used for the control condition because tACS is often accompanied by phosphenes (visual disturbances) and cutaneous sensations under the electrodes, which possibly enables participants to differentiate between active stimulation and the sham condition (Davis et al. 2013). A control condition producing similar side effects is thus needed to ensure that the active and control sessions are as similar as possible. The current study used alpha band tACS because van Driel et al. (2015), who also used theta band stimulation as the active condition and alpha band stimulation as the control condition, reported that theta and alpha tACS resulted in similar side effects. Furthermore, stimulation with higher frequency bands, such as beta frequencies, has been associated with higher phosphene intensity compared with theta band tACS (Turi et al. 2013). Finally, while lapses in sustained attention have been associated in some studies with increased alpha activity, these changes in alpha activity occur over posterior scalp regions [e.g., (O’Connell et al. 2009)], i.e., not over the anterior scalp regions at which we applied tACS. We therefore did not expect midfrontal alpha tACS to directly modulate sustained attention performance.

\section{Data Analysis}

$\mathrm{A}^{\prime}$ and reaction time were calculated as in experiment 1 . Next, data were submitted to repeated measures ANOVAs with the within-subject factors Stimulation Frequency (alpha vs. theta) and Time (pre, during, and post stimulation). Post hoc paired $t$ tests were used when significant results were found. In addition, Wilcoxon signed-rank tests were used to assess whether side effects differed between stimulation frequencies. 
Because, indeed, some self-reported side effects were significantly different (see "Results"), we re-analyzed A' using a linear mixed model, allowing us to include these side effects per stimulation frequency as a covariate. The model tested for main effects of stimulation frequency and time. In addition, it tested for main effects of tingling sensation and light flashes. The model also included a time $\times$ stimulation frequency interaction.

Lastly, a paired samples $t$ test was used to assess whether target line length differed significantly between stimulation frequencies.

\section{Results}

\section{Experiment 1}

We found no significant differences in target line length between the different stimulation groups $(F(94)=2.269, p=$ $0.109, \mathrm{BF}_{01}=1.737$ ), indicating that task difficulty was similar across groups (anodal, cathodal, and sham stimulation).

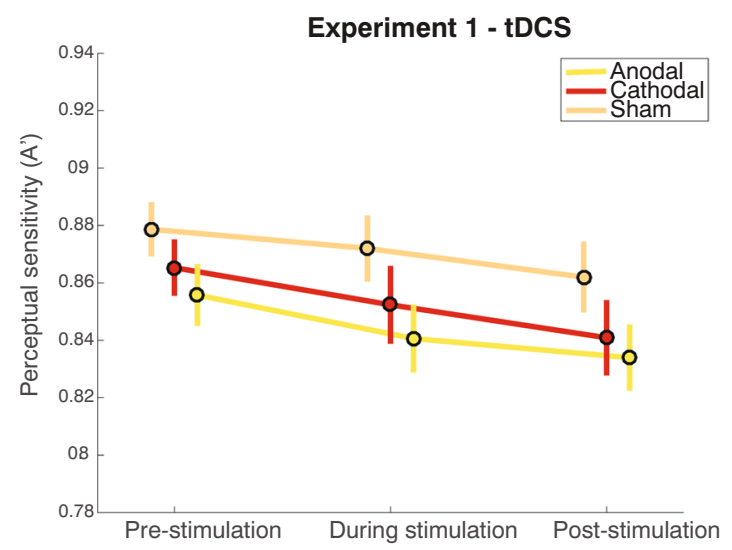

Fig. 2 In both experiments, a robust vigilance decrement was observed, as perceptual sensitivity $\left(\mathrm{A}^{\prime}\right)$ decreased significantly over time. In experiment 1 (left), we found no significant effect of stimulation on the vigilance decrement. In experiment 2 (right), we found a significant Time $\times$ Stimulation frequency effect, such that perceptual sensitivity decreased more over time after theta stimulation compared with alpha stimulation.
In line with previous studies, perceptual sensitivity, as measured by $\mathrm{A}^{\prime}$, declined with time on task (main effect of Time: $\left.F(1.8,167.2)=16.872, p<0.001, \mathrm{BF}_{10}=109,645.144\right)$ (Fig. 2, left). However, there was neither a Time $x$ Stimulation Condition interaction $(F(4,188)=0.325, p=$ $\left.0.861, \mathrm{BF}_{10}=0.029\right)$ nor a main effect of Stimulation Condition $\left(F(2,94)=1.534, p=0.221, \mathrm{BF}_{10}=0.604\right)$. Thus, in contrast to our main prediction, perceptual sensitivity was not modulated by anodal or cathodal tDCS over the mPFC.

For RT, no effects were found (main effect of Time $F(2,188)=1.469, p=0.233, \mathrm{BF}_{10}=0.144$, main effect of Stimulation Condition $\left(F(2,94)=1.161, p=0.318, \mathrm{BF}_{10}=\right.$ $0.520)$, Time $\times$ Stimulation Condition interaction $\left.\left(\mathrm{F}(4,188)=0.681, p=0.606, \mathrm{BF}_{10}=0.056\right)\right)$.

The three different stimulation conditions led to a significant difference in self-reported headache. Specifically, a series of one way ANOVAs showed a main effect of Stimulation Condition on the amount of headache $(F(2,94)=4.926, p=$ $0.009, \mathrm{BF}_{01}=0.215$ ), which was driven by the fact that participants that received anodal stimulation reported a higher amount of headache compared with the other participants (anodal vs. cathodal: $t(41.0)=2.394, p=0.021, \mathrm{BF}_{01}=0.348$;

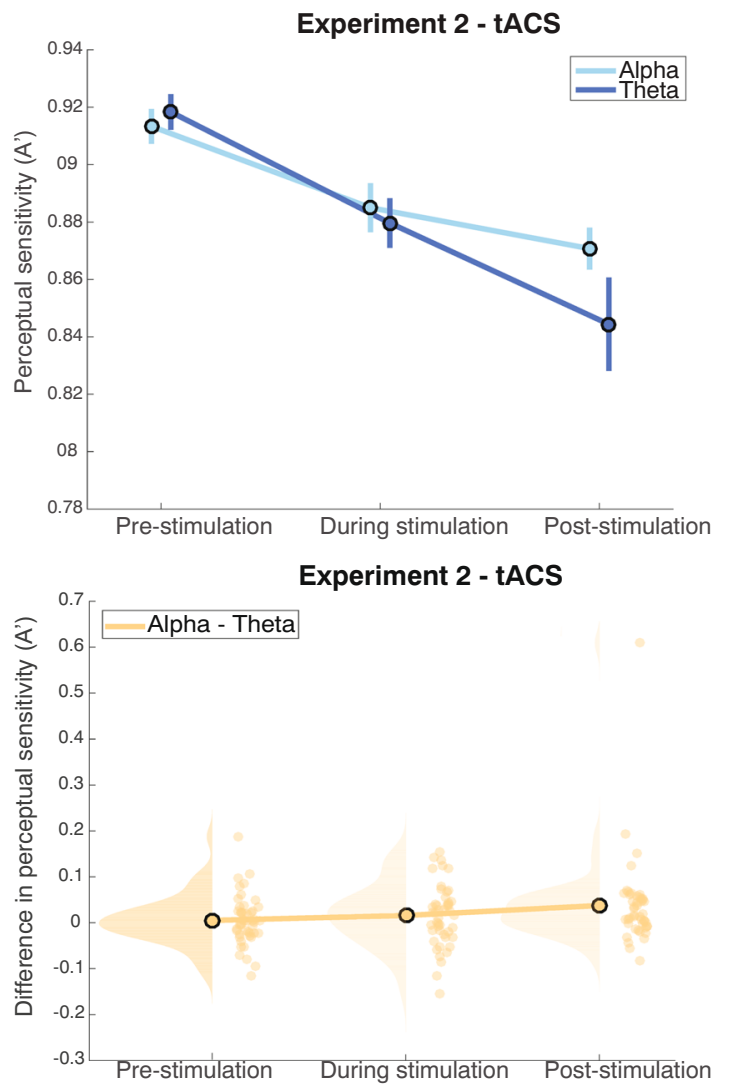

The upper right plot shows the data for alpha and theta stimulation separately. For the lower right plot, we subtracted data from the theta condition from the data from the alpha condition for each individual. Next, we plotted the mean difference as well as the distribution and the individual data points (Allen et al. 2019) 
anodal vs. sham: $t(41.1)=2.356, p=0.023, \mathrm{BF}_{01}=0.457$; sham vs. cathodal: $t(62)=0.062, p=0.951, \mathrm{BF}_{01}=3.903$ ) (median values per stimulation condition: anodal 3 ; cathodal 0 ; sham 0$)$ ) (mean values per stimulation condition: anodal 12.3; cathodal 4.1; sham 4.2). It should be noted that when correcting for multiple comparisons (we ran an ANOVA for each of the ten self-reported side effects), the effect of stimulation condition on headache is not significant. In the anodal group, 12 participants thought their performance worsened during the stimulation block, 11 participants thought their performance remained the same during the stimulation block, and 10 participants thought their performance improved during the stimulation block. For the cathodal group and sham group, the distributions were as follows: cathodal: 13 (improved); 12 (same); 8 (worsened); 1 (data missing), sham: 7 (improved); 13 (same); 10 (worsened); 1 (data missing).

\section{Experiment 2}

We found no significant differences in target line length between the different stimulation frequency sessions $(t(46)=$ $0.264, p=0.793, \mathrm{BF}_{01}=6.110$ ), indicating that task difficulty was similar across conditions.

Again, perceptual sensitivity, as indexed by $\mathrm{A}^{\prime}$, declined with time on task (main effect of Time: $F(1.4,65.9=$ 40.264, $\left.p<0.001, \mathrm{BF}_{10}=3.163 \mathrm{e}+10\right)$. Consistent with our hypothesis, we found a significant Time $\times$ Stimulation Frequency interaction $(F(1.5,71.1)=5.435, p=0.011)$ (Fig. 2 , right). However, in contrast to our prediction, this interaction was driven by the fact that performance declined more in the theta stimulation condition than in the alpha stimulation condition in particular after the stimulation had ended. Yet, the post hoc $t$ test did not reach significance $(t(46)=1.891, p=$ $0.065, \mathrm{BF}_{10}=0.813$ ), although numerically, perceptual sensitivity was lower after theta stimulation than after alpha stimulation. Note also that while the frequentist analysis indicated a significant interaction between Time and Stimulation Frequency, a Bayesian repeated measures ANOVA indicated that the data are 0.565 times less likely under the model that includes the interaction effect $\left(\mathrm{BF}_{10}=6.9 \mathrm{e}+9\right)$ than under the model that only includes the main effects of Time and Stimulation Frequency $\left(\mathrm{BF}_{10}=1.221 \mathrm{e}+10\right)$. Thus, the statistical evidence for a differential effect of stimulation frequency on sustained attention task performance over time was weak. (Note that the outlier in the bottom right plot is not driving the results, but is actually reducing the significance of the effect $(F$ value: 5.4 vs. $5.5 ; p$ value: 0.011 vs. $0.006 ; \mathrm{BF}_{10}=0.565$ vs. $\left.\mathrm{BF}_{10}=0.556\right)$.)

For RT, we found a main effect of Time $(F(1.7$, $\left.78.7)=19.280, p<0.001, \mathrm{BF}_{10}=4993.764\right)$, but no main effect of Stimulation Frequency $(F(1,46)=0.134$, $\left.p=0.716, \mathrm{BF}_{10}=0.149\right)$, nor an interaction $\left(F(1.6,74.7)=1.674, p=0.193, \mathrm{BF}_{10}=0.167\right)$.
In terms of side effects, we also found a difference between the stimulation conditions. Specifically, participants perceived more tingling sensations $\left(Z=-4.053, p<0.001, \mathrm{BF}_{01}=\right.$ $\left.5.341 \mathrm{e}^{-4}\right)$ and more phosphenes $(Z=-4.981, p<0.001$, $\left.\mathrm{BF}_{01}=2.623 \mathrm{e}-7\right)$ during alpha stimulation. This could confound our findings on perceptual sensitivity. In an attempt to control for these potentially confounding side effects, we performed another analysis, in which we tested for a Time $\times$ Stimulation Frequency interaction on perceptual sensitivity using these two side effects as a covariate. Again, we found a main effect of Time $(F(2,230)=33.587, p<0.001)$, indicative of a decrease in performance over time, but now the Time $\times$ Stimulation Frequency interaction did not reach significance $(F(2,230)=2.508, p=0.084)$.

\section{Discussion}

Sustained attention is crucial in many everyday life and professional settings that require continuous monitoring to detect rare and difficult to predict events, such as when driving a car or in air traffic control. In two experiments with relatively large samples sizes ( $n=97$ and $n=47$, respectively), we aimed to improve people's ability to sustain attention over time using transcranial electrical stimulation over mPFC, a key region involved in sustained attention (Langner and Eickhoff 2013). However, in neither experiment, we found convincing evidence that electrical stimulation over the midfrontal cortex can enhance sustained attention. These findings contribute to a growing body of studies that report no effect of electrical brain stimulation on behavior (Horvath et al. 2015; Medina and Cason 2017), but contrast with studies that did observe positive effects of electrical stimulation on sustained attention (Nelson et al. 2014; McIntire et al. 2014). Below, we discuss our findings in greater detail with respect to the current literature, and discuss potential explanations and necessary follow-up studies.

In experiment 1 , neither anodal nor cathodal tDCS over mPFC modulated sustained attention compared with sham tDCS. It is possible that our stimulation did not reach the mPFC. Yet, it has previously been shown that $1 \mathrm{~mA}$ anodal tDCS over midfrontal cortex can increase frontal theta activity, albeit transiently (Miller et al. 2015). Nonetheless, in that study, no effects were observed on a subsequent sustained attention task. Our findings extend this work by showing, using a much larger sample, that midfrontal anodal tDCS concurrent with a sustained attention task is also not associated with performance improvements. In previous studies looking at the effect of tDCS on sustained attention, it was found that tDCS over bilateral dorsolateral prefrontal cortex (DLPFC) could prevent the vigilance decrement (Nelson et al. 2014; McIntire et al. 2014). However, replication of these effects is warranted since some of the effects ascribed to tDCS were 
already apparent before tDCS stimulation started (Nelson et al. 2014). Also, correction to the baseline block, as was applied in these studies, can lead to artificially induced effects, by adding baseline differences to experimental effects. Alternatively, it is possible that we did not choose the most optimal settings in the design of our experiment. We used a different montage than a previous study that reported tDCS effects on sustained attention with bilateral frontal stimulation (although in the presence of baseline differences) (McIntire et al. 2014). Although $1 \mathrm{~mA}$ is a common current strength used for tDCS in attention studies (see for example a recent review by Reteig et al. (2017)), the effects of tDCS may vary as a result of the applied stimulation intensity (Nitsche et al. 2015), though not necessarily in a linear fashion (Batsikadze et al. 2013). Another reason for the null findings could be the fact that we used a between-subject design. tDCS effects are generally subtle and might therefore surface only in a withinsubject design which is more sensitive because it takes out individual variance in baseline task performance and the degree of vigilance decrement over time (although we tried to control for the first with the staircase procedure). However, compared with many tDCS studies that previously reported effects on attentional performance using a between-subject design (Reteig et al. 2017), our sample size was relatively large with 30 to 34 participants in each stimulation group. It should also be noted that the effects of tDCS in general are debated (Horvath et al. 2014). As we pointed out in an earlier review, the wide range in stimulation parameters that is used in tDCS studies of attention and individual differences in the effects of tDCS might contribute to the failure to find (consistent) effects (Reteig et al. 2017). Also, the neural effects of tDCS itself may be smaller than assumed, as it was recently shown that the strength of the electric field in the brain is at the lower bound for it to be physiologically effective (Huang et al. 2017; Vöröslakos et al. 2018). Notably, a recent study that applied cathodal tDCS over left lateral prefrontal cortex found that this increased mind wandering during a sustained attention to response task, but only at $2 \mathrm{~mA}$ (Filmer et al. 2019). Future work is necessary to determine if higher stimulation strength could similarly affect performance on sustained attention tasks that only rarely require a response, as used in our study. To summarize, using $1 \mathrm{~mA}$ anodal tDCS over mPFC, we were not able to improve sustained attention.

In experiment 2 , using a within-subject design with 47 participants, we found effects of tACS on the vigilance decrement. However, we have to be cautious about the interpretation of these results. First, in contrast to our expectations, participants' vigilance decrement became worse after theta stimulation compared with alpha stimulation. As explained in the introduction, we anticipated that theta stimulation would improve performance based on previous studies showing that theta oscillations originating from the mPFC are involved in performance monitoring and task set (re-)energizing
(Cavanagh and Frank 2014). It is possible that we interfered with an already optimally functioning system (in healthy individuals) and therefore found the opposite of what we anticipated. Also, we did not use individually determined theta frequency, but stimulated everyone at $4 \mathrm{~Hz}$. It has been suggested that effects of tACS might be strongest when stimulation happens in sync with the individual intrinsic frequency (Schmidt et al. 2014; Romei et al. 2016). Perhaps by entraining mPFC neurons to a frequency that did not match their intrinsic frequency in some of our participants, we disrupted the system, rather than helped it. Yet, some studies have also reported no effects of midfrontal theta tACS, albeit on a working memory task (Friedrich et al. 2019). Alternatively, it is hence also possible that theta stimulation had no effect, but that alpha stimulation improved performance. Indeed, in a recent study, Clayton et al. found that alpha stimulation over occipital cortex stabilized performance on a visual attention task (2018). The researchers speculated that the alpha stimulation may have prevented top-down reorienting signals from changing the attentional state of the visual system. Alpha stimulation may similarly have stabilized the monitoring state of mPFC. Indeed, sustained attention has not only been associated with oscillations in the theta frequency but also in the alpha and gamma range (Clayton et al. 2015; Makeig and Jung 1995; Braboszcz and Delorme 2011). Frontal alpha-band oscillations may also play a role in cognitive control (e.g., (Hwang et al. 2014)). Yet, without the presence of a sham condition, we cannot dissociate whether the effects that we found are driven by effects in the alpha stimulation condition, the theta stimulation condition, or a combination of both. However, as described in "Materials and methods," including a sham condition has its own disadvantages. Moreover, it should be noted that the results from a Bayesian repeated measures ANOVA did not support the frequentist result of an effect of stimulation frequency on sustained attention task performance over time, as this analysis suggested that the data were almost two times more likely under the model that did not include the interaction effect.

Another difficulty in the interpretation of the obtained results is the fact that we found differences in side effects between the two stimulation frequencies. During alpha stimulation, subjects experienced more tingling sensations and more phosphenes compared with that during theta stimulation. It could be argued that these side effects mainly occurred during the block of stimulation and do not extend to the block after stimulation, in which the difference between the stimulation conditions seems to arise. Also, it seems counterintuitive that an increase in side effects would lead to better rather than worse perceptual sensitivity. One possibility is that thetainduced phosphenes, albeit less frequently experienced, were experienced as much more unpleasant or disruptive than alpha-induced phosphenes. Moreover, we cannot exclude the possibility that experiencing side effects made subjects 
more alert or strongly believe that they were in an active stimulation condition, thereby increasing their motivation to do well. When trying to control for these differences, the Time $\times$ Stimulation Frequency interaction no longer reached significance. This suggests that at least part of the frequencydependent tACS effects on the vigilance decrement can be explained by the differences in side effects between the stimulation frequencies. More work is necessary to better establish at what stimulation intensity side effects, such as phosphenes, may arise at different stimulation frequencies, as they are clearly confounding factors. In sum, although we do find frequency-dependent tACS effects, with the current setup we cannot determine whether participants improved or worsened by the stimulation, and they could simply reflect non-specific side effects. Further research including other control conditions, for example stimulation over another brain region, that evoke similar side effects, is needed to determine the direction of the observed effects and the role of midfrontal theta oscillations in sustained attention.

\section{Conclusions}

To conclude, across the two studies we were not able to find a convincing method to prevent or reduce the vigilant decrement. More research that combines brain stimulation with neuroimaging is needed to determine if and how electrical stimulation over mPFC may affect brain functioning and sustained attention.

Author Contributions MRS, IGS, and HAS conceived and designed the experiments; MRS, IGS, MRG, FG, DK, FSS, and AV collected and analyzed the data; MRS, IGS, and HAS interpreted the data and wrote the manuscript. All authors reviewed the manuscript.

Funding information This research was funded by a Marie Curie European Fellowship grant to MS and a VIDI from the Netherlands Organization for Scientific Research (NWO) to HAS.

Data Availability Data and analysis scripts will be made available after acceptance for publication via the University of Amsterdam Figshare system (https://uvaauas.figshare.com/).

\section{Compliance with Ethical Standards}

Competing Interests The authors declare that they have no competing interests.

Ethics statement The protocol was approved by the Social Sciences ethical committee of the University of Amsterdam. The methods were carried out in accordance with the relevant guidelines and regulations. All participants gave written informed consent in accordance with the Declaration of Helsinki and received course credits or a monetary reward in exchange for participation.
Open Access This article is licensed under a Creative Commons Attribution 4.0 International License, which permits use, sharing, adaptation, distribution and reproduction in any medium or format, as long as you give appropriate credit to the original author(s) and the source, provide a link to the Creative Commons licence, and indicate if changes were made. The images or other third party material in this article are included in the article's Creative Commons licence, unless indicated otherwise in a credit line to the material. If material is not included in the article's Creative Commons licence and your intended use is not permitted by statutory regulation or exceeds the permitted use, you will need to obtain permission directly from the copyright holder. To view a copy of this licence, visit http://creativecommons.org/licenses/by/4.0/.

\section{References}

Allen, M., Poggiali, D., Whitaker, K., Marshall, T. R., \& Kievit, R. A. (2019). Raincloud plots: A multi-platform tool for robust data visualization [version 1; peer review: Awaiting peer review]. Wellcome Open Research, 4.

Batsikadze, G., Moliadze, V., Paulus, W., Kuo, M. F., \& Nitsche, M. A. (2013). Partially non-linear stimulation intensity-dependent effects of direct current stimulation on motor cortex excitability in humans. The Journal of Physiology, 591, 1987-2000. https://doi.org/10. 1113/jphysiol.2012.249730.

Boksem, M. A. S., \& Tops, M. (2008). Mental fatigue: Costs and benefits. Brain Research Reviews, 59, 125-139. https://doi.org/10.1016/ j.brainresrev.2008.07.001.

Boksem, M. A. S., Meijman, T. F., \& Lorist, M. M. (2005). Effects of mental fatigue on attention: An ERP study. Cognitive Brain Research. https://doi.org/10.1016/j.cogbrainres.2005.04.011.

Boksem, M. A. S., Meijman, T. F., \& Lorist, M. M. (2006). Mental fatigue, motivation and action monitoring. Biological Psychology, 72, 123-132. https://doi.org/10.1016/j.biopsycho.2005.08.007.

Bonnefond, A., Doignon-Camus, N., Hoeft, A., \& Dufour, A. (2011). Impact of motivation on cognitive control in the context of vigilance lowering: An ERP study. Brain and Cognition, 77, 464-471. https:// doi.org/10.1016/j.bandc.2011.08.010

Braboszcz, C., \& Delorme, A. (2011). Lost in thoughts: Neural markers of low alertness during mind wandering. Neuroimage. https://doi. org/10.1016/j.neuroimage.2010.10.008.

Cavanagh, J. F., \& Frank, M. J. (2014). Frontal theta as a mechanism for cognitive control. Trends in Cognitive Sciences, 1-8. https://doi.org/ 10.1016/j.tics.2014.04.012.

Christie, S. T., \& Schrater, P. (2015). Cognitive cost as dynamic allocation of energetic resources. Frontiers in Neuroscience, 9, 1-15. https://doi.org/10.3389/fnins.2015.00289.

Clayton, M. S., Yeung, N., \& Cohen, K. R. (2015). The roles of cortical oscillations in sustained attention. TiCS, 1-8. https://doi.org/10. 1016/j.tics.2015.02.004.

Clayton, M. S., Yeung, N., Kadosh, R. C., Clayton, M. S., Yeung, N., \& Kadosh, R. C. (2018). Performance on visual attention tasks electrical stimulation of alpha oscillations stabilizes performance on visual attention tasks. Journal of Experimental Psychology. General. https://doi.org/10.1037/xge0000502.

Davis, N. J., Gold, E., Pascual-Leone, A., \& Bracewell, R. M. (2013). Challenges of proper placebo control for non-invasive brain stimulation in clinical and experimental applications. The European Journal of Neuroscience, 38, 2973-2977. https://doi.org/10.1111/ ejn.12307.

Filmer, H. L., Griffen, A., \& Dux, P. E. (2019). For a minute there, I lost myself...dosage dependent increases in mind wandering via 
prefrontal tDCS. Neuropsychologia. https://doi.org/10.1016/j. neuropsychologia.2019.04.013.

Friedrich, E. V. C., Berger, B., Minarik, T., Schmid, D., Peylo, C., \& Sauseng, P. (2019). No enhancing effect of fronto-medial tDCS on working memory processes. Journal of Cognitive Enhancement, 3. https://doi.org/10.1007/s41465-019-00136-5.

Grier, R. A., Warm, J. S., Dember, W. N., Matthews, G., Galinsky, T. L., Szalma, J. L., et al. (2003). The vigilance decrement reflects limitations in effortful attention, not mindlessness. Human Factors: The Journal of the Human Factors and Ergonomics Society, 45, 349359. https://doi.org/10.1518/hfes.45.3.349.27253.

Helton, W. S., \& Warm, J. S. (2008). Signal salience and the mindlessness theory of vigilance. Acta Psychologica, 129, 18-25. https://doi. org/10.1016/j.actpsy.2008.04.002.

Herrmann, C. S., Rach, S., Neuling, T., \& Strüber, D. (2013). Transcranial alternating current stimulation: A review of the underlying mechanisms and modulation of cognitive processes. Frontiers in Human Neuroscience, 7, 279. https://doi.org/10.3389/fnhum. 2013.00279.

Hopstaken, J. F., van der Linden, D., Bakker, A. B., \& Kompier, M. A. J. (2015). A multifaceted investigation of the link between mental fatigue and task disengagement. Psychophysiology, 52, 305-315. https://doi.org/10.1111/psyp.12339.

Horvath, J. C., Forte, J. D., \& Carter, O. (2014). Evidence that transcranial direct current stimulation (tDCS) generates little-to-no reliable neurophysiologic effect beyond MEP amplitude modulation in healthy human subjects: A systematic review. Neuropsychologia. https://doi.org/10.1016/j.neuropsychologia.2014.11.021.

Horvath, J. C., Forte, J. D., \& Carter, O. (2015). Quantitative review finds no evidence of cognitive effects in healthy populations from singlesession transcranial direct current stimulation (tDCS). Brain Stimulation, 8, 535-550. https://doi.org/10.1016/j.brs.2015.01.400.

Huang, Y., Liu, A. A., Lafon, B., Friedman, D., Dayan, M., Wang, X., et al. (2017). Measurements and models of electric fields in the in vivo human brain during transcranial electric stimulation. Elife, 6, e18834. https://doi.org/10.7554/eLife.18834.

Hwang, K., Ghuman, A. S., Manoach, D. S., Jones, S. R., \& Luna, B. (2014). Cortical neurodynamics of inhibitory control. The Journal of Neuroscience. https://doi.org/10.1523/JNEUROSCI.4889-13.2014.

Langner, R., \& Eickhoff, S. B. (2013). Sustaining attention to simple tasks: A meta-analytic review of the neural mechanisms of vigilant attention. Psychological Bulletin, 139, 870-900. https://doi.org/10. 1037/a0030694.

Lorist, M. M., Bezdan, E., ten Caat, M., Span, M. M., Roerdink, J. B. T. M., \& Maurits, N. M. (2009). The influence of mental fatigue and motivation on neural network dynamics; an EEG coherence study. Brain Research, 1270, 95-106. https://doi.org/10.1016/j.brainres. 2009.03.015.

MacLean, K. A., Aichele, S. R., Bridwell, D. A., Mangun, G. R., Wojciulik, E., \& Saron, C. D. (2009). Interactions between endogenous and exogenous attention during vigilance. Attention, Perception, Psychophys, 71, 1461-1467. https://doi.org/10.3758/ APP.

Makeig, S., \& Jung, T. P. (1995). Changes in alertness are a principal component of variance in the EEG spectrum. Neuroreport. https:// doi.org/10.1097/00001756-199512000-00051.

McIntire, L. K., McKinley, R. A., Goodyear, C., \& Nelson, J. (2014). A comparison of the effects of transcranial direct current stimulation and caffeine on vigilance and cognitive performance during extended wakefulness. Brain Stimulation, 7, 499-507. https://doi.org/10. 1016/j.brs.2014.04.008.

Medina, J., \& Cason, S. (2017). No evidential value in samples of transcranial direct current stimulation (tDCS) studies of cognition and working memory in healthy populations. Cortex, 94, 131-141. https://doi.org/10.1016/j.cortex.2017.06.021.
Miller, J., Berger, B., \& Sauseng, P. (2015). Anodal transcranial direct current stimulation (tDCS) increases frontal-midline theta activity in the human EEG: A preliminary investigation of non-invasive stimulation. Neuroscience Letters, 588. https://doi.org/10.1016/j.neulet. 2015.01.014.

Nelson, J. T., McKinley, R. A., Golob, E. J., Warm, J. S., \& Parasuraman, R. (2014). Enhancing vigilance in operators with prefrontal cortex transcranial direct current stimulation (tDCS). Neuroimage, 85, 909-917. https://doi.org/10.1016/j.neuroimage.2012.11.061.

Nitsche, M. A., \& Paulus, W. (2011). Transcranial direct current stimulation-update 2011. Restorative Neurology and Neuroscience, 29, 463-492. https://doi.org/10.3233/RNN-20110618.

Nitsche, M. A., Kuo, M., Paulus, W., Antal, A. (2015). Textbook of Neuromodulation. https://doi.org/10.1007/978-1-4939-1408-1.

Norman, D. .A, Shallice, T. (1986). Attention to action: Willed and automatic control of behaviour. Conscious. self-regulation. https://doi. org/10.1098/rstb.1996.0132.

O'Connell, R. G., Dockree, P. M., Robertson, I. H., Bellgrove, M. A., Foxe, J. J., \& Kelly, S. P. (2009). Uncovering the neural signature of lapsing attention: Electrophysiological signals predict errors up to $20 \mathrm{~s}$ before they occur. The Journal of Neuroscience, 29, 8604 8611. https://doi.org/10.1523/jneurosci.5967-08.2009.

Reteig, L. C., Talsma, L. J., van Schouwenburg, M. R., \& Slagter, H. A. (2017). Transcranial electrical stimulation as a tool to enhance attention. Journal of Cognitive Enhancement, 1, 10-25. https://doi. org/10.1007/s41465-017-0010-y.

Reteig, L. C., van den Brink, R. L., Prinssen, S., Cohen, M. X., \& Slagter, H. A. (2019). Sustaining attention for a prolonged period of time increases temporal variability in cortical responses. Cortex, 117, 1632. https://doi.org/10.1016/j.cortex.2019.02.016.

Ridderinkhof, K. R., Ullsperger, M., Crone, E. A., \& Nieuwenhuis, S. (2004). The role of the medial frontal cortex in cognitive control. Science (80- ), 306, 443-447. https://doi.org/10.1126/science. 1100301.

Robert, G., \& Hockey, J. (1997). Compensatory control in the regulation of human performance under stress and high workload: A cognitiveenergetical framework. Biological Psychology. https://doi.org/10. 1016/S0301-0511(96)05223-4.

Robertson, I. H., \& O'Connell, R. G. (2010). Vigilant attention. In A. C. Nobre \& J. T. Coull (Eds.), Atten. time (pp. 79-88). Oxford: Oxford University Press.

Robertson, I. H., Manly, T., Andrade, J., Baddeley, B. T., \& Yiend, J. (1997). "Oops!": Performance correlates of everyday attentional failures in traumatic brain injured and normal subjects. Neuropsychologia. https://doi.org/10.1016/S0028-3932(97)000158.

Romei, V., Bauer, M., Brooks, J. L., Economides, M., Penny, W., Thut, G., et al. (2016). Causal evidence that intrinsic beta-frequency is relevant for enhanced signal propagation in the motor system as shown through rhythmic TMS. Neuroimage, 126, 120-130. https://doi.org/10.1016/j.neuroimage.2015.11.020.

Schmidt, S. L., Iyengar, A. K., Foulser, A. A., Boyle, M. R., \& Fröhlich, F. (2014). Endogenous cortical oscillations constrain neuromodulation by weak electric fields. Brain Stimulation, 7, 878-889. https://doi.org/10.1016/j.brs.2014.07.033.

Slagter, H. A., Prinssen, S., Reteig, L. C., \& Mazaheri, A. (2016). Facilitation and inhibition in attention: Functional dissociation of pre-stimulus alpha activity, P1, and N1 components. Neuroimage, 125, 25-35. https://doi.org/10.1016/j.neuroimage.2015.09.058.

Smallwood, J., \& Schooler, J. W. (2006). The restless mind. Psychological Bulletin. https://doi.org/10.1037/0033-2909.132.6. 946.

Stanislaw, H., \& Todorov, N. (1999). Calculation of signal detection theory measures. Behav Res Methods, Instruments, Comput, 31, $137-149$. 
Stuss, D. T., Shallice, T., Alexander, M. P., \& Picton, T. W. (1995). A multidisciplinary approach to anterior attentional functions. Annals of the New York Academy of Sciences, 769, 191-212. https://doi.org/ 10.1111/j.1749-6632.1995.tb38140.x.

Taylor, M. M., \& Creelman, C. D. (1967). PEST: Efficient estimates on probability functions. The Journal of the Acoustical Society of America, 41, 782-787. https://doi.org/10.1121/1.1910407.

Team, J. (2019). JASP (Version 0.10.2) [Computer software].

Turi, Z., Ambrus, G. G., Janacsek, K., Emmert, K., Hahn, L., Paulus, W., et al. (2013). Both the cutaneous sensation and phosphene perception are modulated in a frequency-specific manner during transcranial alternating current stimulation. Restorative Neurology and Neuroscience, 31, 275-285. https://doi.org/10.3233/RNN-120297.

van Driel, J., Sligte, I. G., Linders, J., Elport, D., \& Cohen, M. X. (2015). Frequency band-specific electrical brain stimulation modulates cognitive control processes. PLoS One, 10, e0138984. https://doi.org/ 10.1371/journal.pone.0138984.
Vöröslakos, M., Takeuchi, Y., Brinyiczki, K., Zombori, T., Oliva, A., Fernández-Ruiz, A., et al. (2018). Direct effects of transcranial electric stimulation on brain circuits in rats and humans. Nature Communications, 9. https://doi.org/10.1038/s41467-018-02928-3.

Warm, J. S., Parasuraman, R., \& Matthews, G. (2008). Vigilance requires hard mental work and is stressful. Hum Factors J Hum Factors Ergon Soc, 50, 433-441. https://doi.org/10.1518/ $001872008 X 312152$.

Wascher, E., Rasch, B., Sänger, J., Hoffmann, S., Schneider, D., Rinkenauer, G., et al. (2014). Frontal theta activity reflects distinct aspects of mental fatigue. Biological Psychology, 96, 57-65. https:// doi.org/10.1016/j.biopsycho.2013.11.010.

Publisher's Note Springer Nature remains neutral with regard to jurisdictional claims in published maps and institutional affiliations. 